\title{
Adipocytes regulate the bone marrow microenvironment in a mouse model of obesity
}

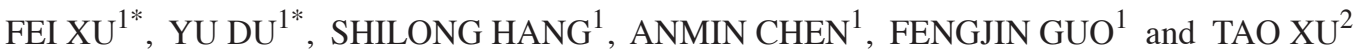 \\ Departments of ${ }^{1}$ Orthopedics and ${ }^{2}$ Rehabilitation, Tongji Hospital, Tongji Medical College, \\ Huazhong University of Science and Technology, Wuhan, Hubei 430030, P.R. China
}

Received October 15, 2012; Accepted January 14, 2013

DOI: $10.3892 / \mathrm{mmr} .2013 .1572$

\begin{abstract}
Obesity is markedly associated with abnormal bone density indicating the importance of adipocytes in bone metabolism. However, the specific function of adipocytes remains unclear, with marked discrepancies in observations of previous studies. In the present study, the effect of adipocytes on osteoblasts/osteoclasts was analyzed. A mouse model of obesity was established and an in vitro co-culture system was utilized containing adipocyte and MC3T3/RAW 264.7 cells in a Transwell plate. Compared with control mice, obese mice exhibited low body weight and bone mineral density of the tibia and fat cells were observed to accumulate in bone marrow. MC3T3/RAW 264.7 cells were co-cultured with adipocytes and the mRNA and protein expression of alkaline phosphatase and osteocalcin was found to be decreased in MC3T3-E1 cells and mRNA and protein expression of tartrate-resistant acid phosphatase and cathepsin K was significantly increased in RAW 264.7 cells. In addition, the effect of adipocytes on the osteoprotegerin (OPG)/receptor activator of nuclear factor $\kappa \mathrm{B}$ ligand (RANKL)/RANK system indicated that the RANKL/OPG ratio secreted by osteoblasts increased and RANK expression by osteoclasts increased, leading to increased osteoclastogenesis. These results indicate that bone metabolism is impaired in obese mice leading to decreased
\end{abstract}

Correspondence to: Professor Tao Xu, Department of Rehabilitation, Tongji Hospital, Tongji Medical College, Huazhong University of Science and Technology, 1095 Jiefang Road, Wuhan, Hubei 430030, P.R. China

E-mail: skyfrost444@gmail.com

${ }^{*}$ Contributed equally

Abbreviations: TRAP, tartrate-resistant acid phosphatase; RANK, receptor activator of nuclear factor $\kappa \mathrm{B}$; RANKL, receptor activator of nuclear factor $\kappa \mathrm{B}$ ligand; OPG, osteoprotegerin; CTSK, cathepsin K

Key words: adipocyte, osteoblasts osteoclast, obesity, osteoprotegerin/receptor activator of nuclear factor $\kappa \mathrm{B}$ ligand/receptor activator of nuclear factor $\kappa \mathrm{B}$ system osteoblastogenesis and marked increases in osteoclastogenesis and low bone mass.

\section{Introduction}

Obesity is becoming increasingly prevalent, leading to reduced life expectancy and increased health problems (1). High body mass index (BMI) is associated with various diseases, including cardiovascular, obstructive sleep apnea, diabetes mellitus type 2, specific types of cancer and osteoarthritis (2). Bone metabolism is also abnormal in obesity (3) and positive and negative factors have been identified to be associated with bone health status (4-6). A number of previous studies have revealed that obesity stimulates bone formation by inhibiting apoptosis. A recent study demonstrated that high BMI prevents normal bone fracture and osteoporotic fracture in various age groups and genders (7). By contrast, it has also been reported that osteoporosis occurs in obese individuals (8). These inconsistencies may be due to the use of different animal models in each study.

Adipose tissue, a large endocrine organ, secretes a number of hormones that affect bone metabolism. Adipocytes in the bone marrow microenvironment differentiate from bone marrow mesenchymal cells and secrete cytokines which affect osteoblast and osteoclast levels in bone marrow. The effect of adipocytes on osteoblasts/osteoclasts remains controversial.

The aim of the present study was to identify the function of adipocytes in the bone marrow microenvironment. To analyze the biological foundations of bone metastasis in obesity, a high-fat diet was selected to establish a mouse model of obesity (9). The results indicate that adipocytes accumulate in the bone marrow environment and affect bone turnover and osteoblast/osteoclast differentiation.

\section{Materials and methods}

Chemicals and proteins. Recombinant murine receptor activator of nuclear factor $\kappa \mathrm{B}$ ligand (RANKL) was purchased from R\&D Systems (Minneapolis, MN, USA). Antibodies were purchased from Cell Signaling Technology, Inc. (Danvers, MA, USA) and Abcam (Cambridge, UK). All other chemicals were from Sigma-Aldrich (St. Louis, MO, USA). Osteoprotegerin (OPG), RANKL and RANK ELISA kits were obtained from R\&D Systems. Alkaline phosphatase (ALP) 
and tartrate-resistant acid phosphatase (TRAP) staining kits were purchased from Sigma-Aldrich.

Animals. Male C57BL/6 mice were purchased from Beijing HFK Bio-technology Co., Ltd. (Beijing, China) at 7-8 weeks old. Animals were randomly divided into 2 groups. One group was housed in a 12-h dark/light cycle and fed high-fat food and water ad libitum, the other group was fed normal food (control). All procedures were approved by the Animal Care and Use Committee, Tongji Medical College (Wuhan, China)

Animals were sacrificed by $\mathrm{CO}_{2}$ inhalation and cervical dislocation 12 weeks later. Weights of bilateral inguinal fat and the right tibiae were recorded. The right tibiae were analyzed using micro-computed tomography (mCT). The left tibiae were embedded undecalcified in methylmethacrylate and analyzed by histomorphometry.

mCT analysis. mCT analysis was performed using a Scanco $\mu$ CT50 (Scanco Medical AG, Bassersdorf, Switzerland). Scans were performed using the following instrument settings: $\mathrm{E}$, $70 \mathrm{KVp}$; I, $110 \mu \mathrm{A}$, increment, $10 \mu \mathrm{m}$; threshold value, 289. Parameters of the tibia were computed by the software in $\mu \mathrm{CT} 50$.

Cell culture. The murine preosteoblastic cell line, MC3T3-E1, was purchased from the American Type Culture Collection (ATCC; Manassas, VA, USA). Murine bone marrow stromal cells (BMSCs) were purchased from Cyagen (Guangzhou, China). RAW 264.7 murine pre-osteoclastic cells were purchased from ATCC. The cells were cultured in $\alpha$-MEM (Hyclone Laboratories, Inc., Logan, UT, USA) supplemented with $100 \mathrm{U} / \mathrm{ml}$ penicillin, $100 \mu \mathrm{g} / \mathrm{ml}$ streptomycin and $10 \% \mathrm{FCS}$ (Gibco-BRL, Carlsbad, CA, USA) at $37^{\circ} \mathrm{C}$ in a humidified atmosphere of $5 \% \mathrm{CO}_{2}$. The medium was changed every 3 days.

For adipocyte differentiation, BMSCs were cultured in $\alpha$-MEM supplemented with $10 \% \mathrm{FCS}, 100 \mathrm{U} / \mathrm{ml}$ penicillin and $100 \mathrm{mg} / \mathrm{ml}$ streptomycin in a $5 \% \mathrm{CO}_{2}$ humidified atmosphere and allowed to reach confluence. After 2 days of confluence, BMSCs were induced by exposure to a differentiation medium containing $10.0 \mu \mathrm{g} / \mathrm{ml}$ insulin, $1 \mu \mathrm{M}$ dexamethasone and $0.5 \mathrm{mM}$ 3-iso-butyl-1-methylxanthine in $10 \%$ FBS-supplemented $\alpha$-MEM for $48 \mathrm{~h}$. Following incubation, the culture medium was changed to $\alpha$-MEM supplemented with $10 \% \mathrm{FCS}, 10.0 \mu \mathrm{g} / \mathrm{ml}$ insulin for an additional $48 \mathrm{~h}$. Cells were cultured in $\alpha$-MEM supplemented with $10 \% \mathrm{FCS}, 100 \mathrm{U} / \mathrm{ml}$ penicillin and $100 \mathrm{mg} / \mathrm{ml}$ streptomycin for 4 days. The cells were then harvested and used for co-culture and detected by Oil Red staining (Fig. 2A).

Osteoblast differentiation. A Transwell co-culture system was used to analyze osteoblast differentiation. MC3T3-E1 cells $\left(5 \times 10^{6}\right.$ cells/well $)$ were cultured in 6 -well plates (Corning, Tewksbury, MA, USA) until $80 \%$ confluence was reached after 2 days. MC3T3-E1 cells were single- and co-cultured. For co-culture, following successful induction of adipocytes in the upper chambers of the transwell plate, the upper chamber was inserted into the 6-well plate with MC3T3-E1 cells. Following $24 \mathrm{~h}$, the culture medium was replaced with condition medium ( $\alpha$-MEM containing $10 \%$ FCS, $10 \mathrm{mM} \beta$-glycerophosphate, $50 \mu \mathrm{g} / \mathrm{ml} \mathrm{L}$-ascorbic acid and $100 \mathrm{nM}$ dexamethasone) for 7 days. The medium was changed every 2 days. For the last $24 \mathrm{~h}$ prior to harvesting, cells were grown in $2 \mathrm{ml}$ condition medium without FCS and the medium was collected for ELISA analysis. Cells in the lower chambers were detected using ALP staining and activities.

Osteoclast differentiation. For osteoclast differentiation studies, pre-osteoclast RAW 264.7 cells were used. Pre-osteoclast differentiation was performed as described for preosteoblast differentiation, however, the condition medium contained $10 \% \mathrm{FCS}$ and $10 \mathrm{ng} / \mathrm{ml}$ RANKL supplemented $\alpha$-MEM. Harvested cells were analyzed by TRAP staining and activities.

OPG and RANKL ELISA. OPG and RANKL levels were analyzed in the osteoblast differentiation medium using a commercially available ELISA kit according to the manufacturer's instructions. Each sample was analyzed three times.

RNA isolation and real-time PCR analysis. Total RNA was isolated from harvested cells using TRIzol reagent (Invitrogen Life Technologies, Carlsbad, CA, USA). Extracted RNA was transcribed into cDNA using a cDNA RT kit (Invitrogen Life Technologies) according to the manufacturer's instructions. Gene expression analysis was performed using real-time PCR (iQ5; Bio-Rad, Hercules, CA, USA) and normalized against $\beta$-actin. In the present study, runt-related transcription factor 2 (RUNX2), ALP, osteocalcin, OPG and RANKL expression in osteoblasts and RANK, cathepsin K (CTSK) and TRAP expression in osteoclasts was detected.

Western blot analysis. Total cell lysates were obtained by lysing cells in RIPA buffer containing $50 \mathrm{mM}$ Tris- $\mathrm{HCl}$, $150 \mathrm{mM} \mathrm{NaCl}, 1 \% \mathrm{NP}-40,0,1 \%$ SDS, $0,5 \%$ sodium deoxycholate, $2 \mathrm{mM}$ sodium fluoride, $1 \mathrm{mM}$ EDTA, $1 \mathrm{mM}$ EGTA and protease inhibitor cocktail. Protein concentration was determined using the bicinchoninic acid protein assay (Pierce Biotechnology, Inc., Rockford, IL, USA). Proteins were separated by SDS-PAGE, transferred to nitrocellulose, blocked and incubated with primary antibodies. The membrane was washed and incubated with the respective secondary antibodies conjugated with peroxidase. Protein detection was performed using a chemiluminescence detection system (Pierce Biotechnology, Inc.). RUNX2, bone alkaline phosphatase, osteocalcin, OPG and RANKL in osteoblasts and RANK and CTSK protein expression in osteoclasts was detected.

Statistical analysis. Data were presented as the mean \pm SEM. Statistical analysis was performed using the Student's t-test. Experiments were repeated three times. $\mathrm{P}<0.05$ was considered to indicate a statistically significant difference.

\section{Results}

Obese mice have lower tibial bone mineral density. Tibial weight in obese mice was lower than that of the control (Fig. 1A). Micro-CT revealed that bone mineralization density of tibiae in obese mice was lower compared with the control (Fig. 1B and Table I). 

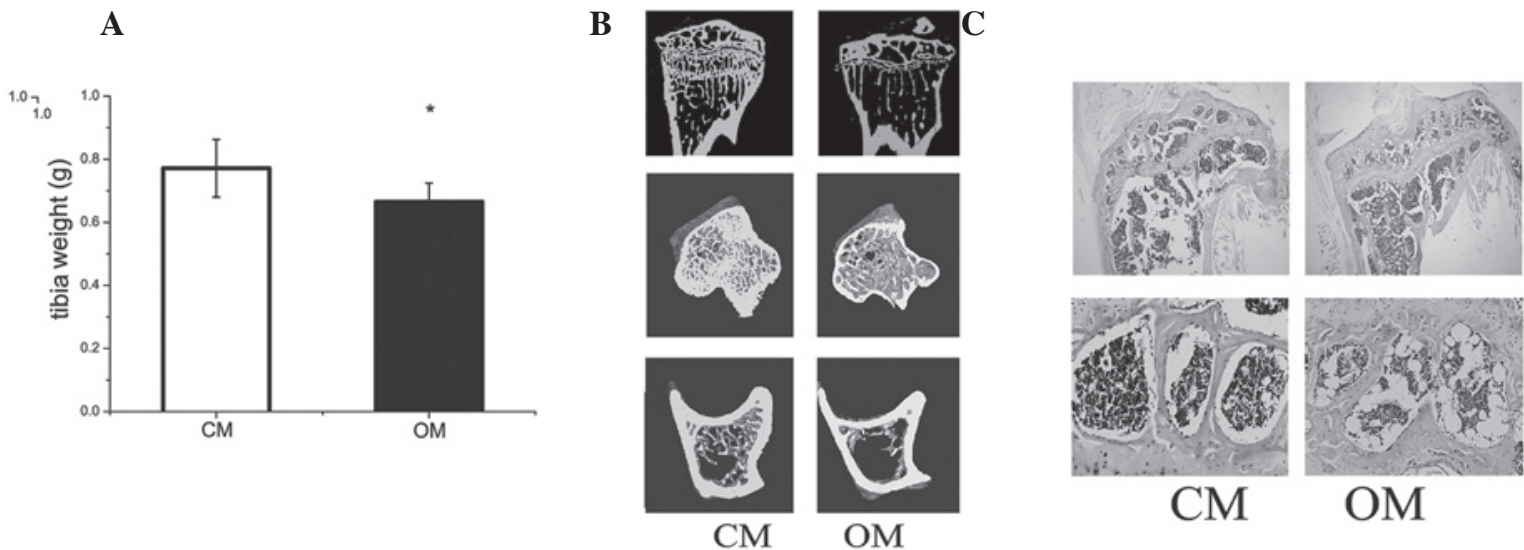

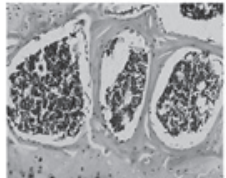

$\mathrm{CM}$

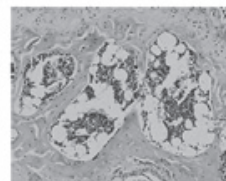

$\mathrm{OM}$

Figure 1. High-fat diet mice exhibited increased tibia weight and bone mineral density, compared with normal diet mice. (A) Tibia weight (g), (B) mCT of the tibia, representative three-dimensiomal images of proximal and middle tibia, (C) HE staining of tibiae revealed accumulated adipocytes in the tibia of obese mice compared with the control (magnification: upper images, $\mathrm{x} 4$; lower images, $\mathrm{x} 10$ ). mCT, micro-computed tomography.

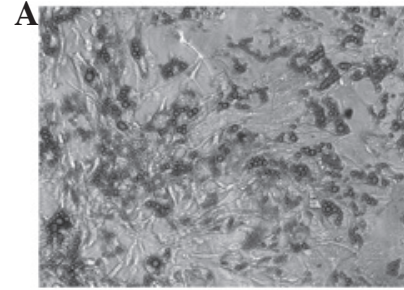

$\mathbf{B}$
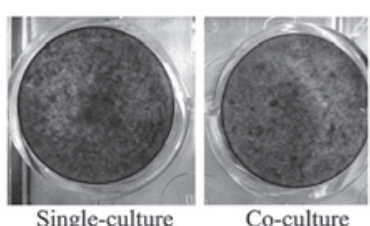

D

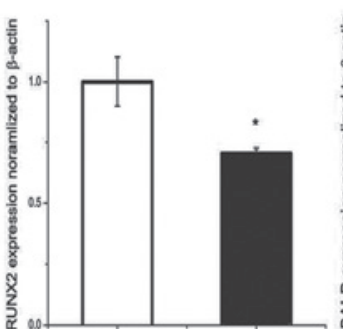

C

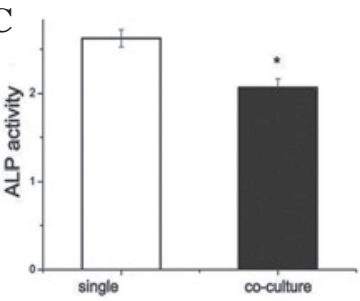

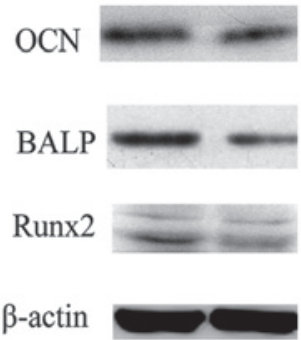

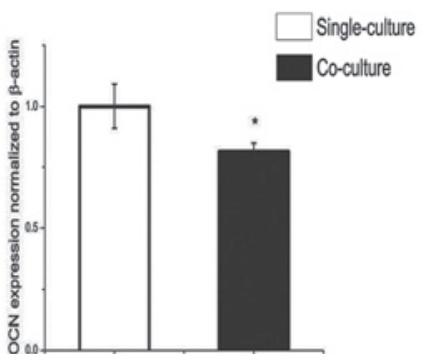

Figure 2. Osteoblast differentiation affected by adipocytes. (A) Oil red staining of adipocytes derived from BMSCs. (B and C) ALP staining and activity of osteoblasts in single- and co-culture with adipocytes. (D) Real-time PCR analysis of gene expression in osteoblasts. (E) Western blot analysis of OCN, BALF, RUNX2 protein expression in osteoblasts. " $\mathrm{P}<0.05$. BMSCs, bone marrow stromal cells. OCN; osteocalcin; BALP, bone alkaline phosphatase; RUNX2, runt-related transcription factor 2.

Histomorphometric analysis indicated an increased number of adipocytes in the bone marrow of obese mice compared with the control (Fig. 1C).

Differentiation of preosteoblasts is decreased by co-culture with adipocytes. In the co-culture system, adipocytes from fully differentiated BMSCs were observed to decrease mRNA and protein expression of ALP (30\%) and osteocalcin (OCN; $10 \%$ ) in osteoblasts at day 5 of differentiation (Fig. 2D).

BMSCs decreased mRNA levels of ALP and OCN in osteoblasts at day 5 of differentiation. These results are consistent with protein levels of these genes (Fig. 2E).
To determine whether adipocytes had any effect on preosteoblast differentiation, ALP staining (Fig. 2B) and analysis of activity were conducted. Co-culture for 7 days was found to significantly reduce ALP activity by $34 \%$ compared with the single-culture group (Fig. 2C).

Differentiation of pre-osteoclasts is increased by co-culture with adipocytes. Since obese mice exhibit accumulation of adipocytes in the bone marrow microenvironment, the effect of adipocytes on osteoclastogenesis and osteoclast-specific gene expression by RANKL-induced osteoclast differentiation was analyzed. Following co-culture with adipocytes, 


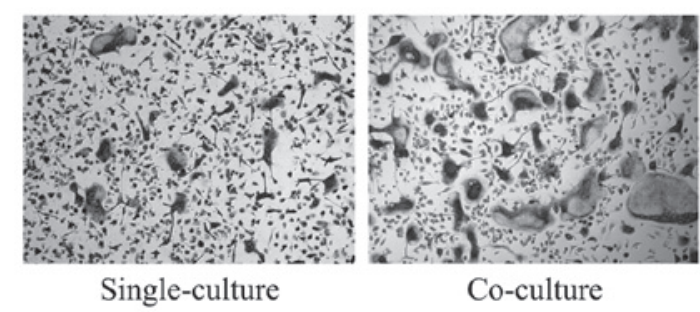

B

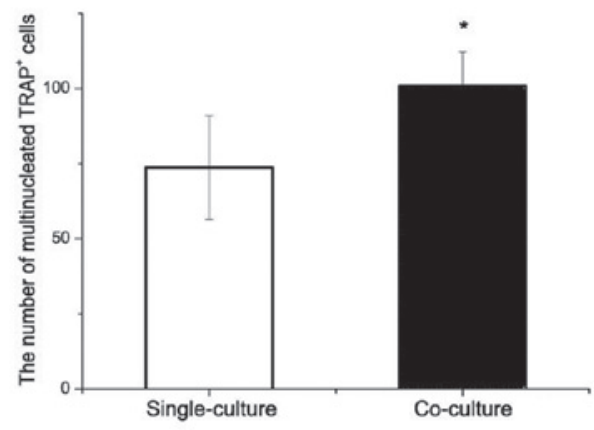

C

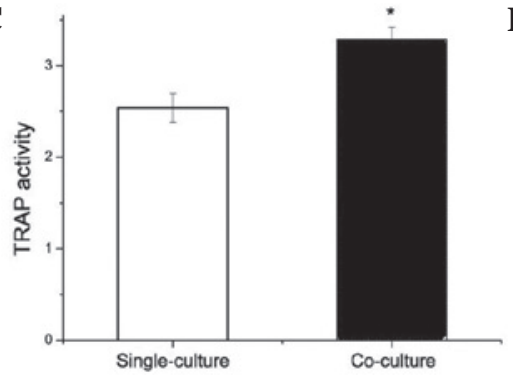

D

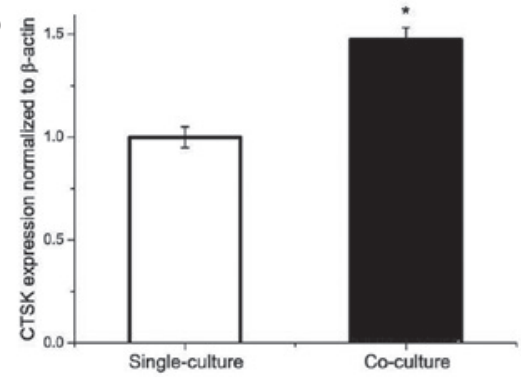
CTSK

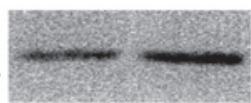

$\beta$-actin

Figure 3. Osteoclast differentiation of RAW 264.7 cells in the presence of RANKL (10 ng/ml) affected by adipocytes. (A) Appearance of multinucleated TRAP-positive osteoclastic cells developed in single-culture and co-culture with adipocyte. (B and C) The number of multinucleated TRAP-positive cells and TRAP activity detected in the two groups. (D) Real-time PCR analysis of gene expression in osteoclasts. (E) Western blot analysis of CTSK protein expression in osteoclasts. " $\mathrm{P}<0.05$, vs. single-culture. TRAP, tartrate-resistant acid phosphatase; CTSK, cathepsin K; RANKL, receptor activator of nuclear factor $\kappa \mathrm{B}$ ligand.

A
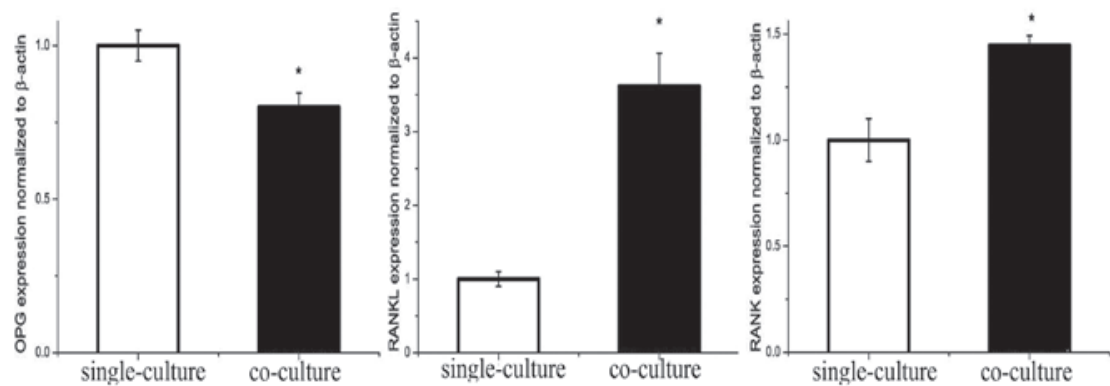

B
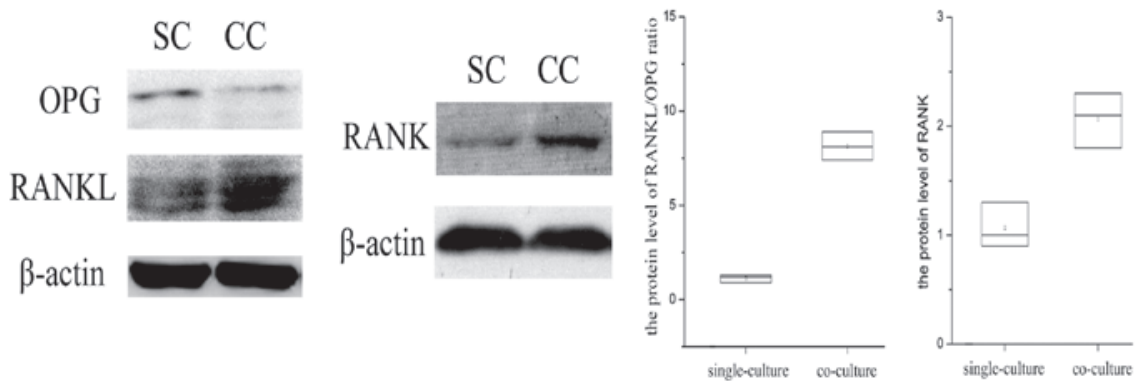

Figure 4. OPG, RANKL and RANK expression in osteoblasts and osteoclasts was altered by adipocytes. (A) Gene expression detected by real-time PCR. (B) Protein expression detected by western blot analysis. The RANKL/OPG ratio in osteoblasts was significantly increased ( $\sim$-fold) in the CC group compared with SC, and RANK expression in osteoclasts was increased in the CC group. The experiment was repeated three times. Vertical bars represent SD. "P $<0.05$, vs. single-culture. OPG, osteoprotegerin; RANKL, receptor activator of nuclear factor $\mathrm{\kappa B}$ ligand; CC, co-culture; SC, single-culture.

osteoclastogenesis was increased compared with that of single-cultured samples (Fig. 3A). The number of multinucleated TRAP-positive cells was increased by $35 \%$ (Fig. 3b) and the TRAP activity was $40 \%$ higher in the co-culture compared with single-culture (Fig. 3C).

Gene expression analysis revealed that adipocytes affect CTSK expression (Fig. 3D). CTSK protein expression in osteo- clasts was increased by co-culture with adipocytes (Fig. 3E). The results indicate that adipocytes may increase osteoclastogenesis in vitro.

OPG/RANKL/RANK trail levels in osteoblasts and osteoclasts are altered by co-culture with adipocytes. OPG/ RANKL/RANK is an essential mechanism for regulation 
Table I. BMD of cortical bone mass in control and obese mice.

\begin{tabular}{cccc}
\hline & $\begin{array}{c}\text { C57BL/6 } \\
(\mathrm{n}=4)\end{array}$ & $\begin{array}{c}\text { Obese mice } \\
(\mathrm{n}=4)\end{array}$ & P-value \\
\hline $\mathrm{BMD}(\mathrm{mg} \mathrm{HA} / \mathrm{ccm})$ & $1342.7 \pm 108.2$ & $706.3 \pm 94.1^{\mathrm{a}}$ & 0.006
\end{tabular}

${ }^{a} \mathrm{P}<0.01$, vs. control. BMD, bone mineral density.

Table II. OPG and RANKL levels in the culture medium of the osteoblastogenesis assay, detected by ELISA.

\begin{tabular}{lccr}
\hline & $\begin{array}{c}\text { Single-culture } \\
(\mathrm{pg} / \mathrm{ml}, \mathrm{n}=6)\end{array}$ & $\begin{array}{c}\text { Co-culture } \\
(\mathrm{pg} / \mathrm{ml}, \mathrm{n}=6)\end{array}$ & P-value \\
\hline OPG & $1342.7 \pm 208.2$ & $706.3 \pm 94.1^{\mathrm{a}}$ & 0.004 \\
RANKL & $1439.7 \pm 143.5$ & $5471.2 \pm 432.5^{\mathrm{a}}$ & 0.009 \\
\hline
\end{tabular}

${ }^{\mathrm{a} P}<0.01$, vs. single-culture. OPG, osteoprotegerin; RANKL, receptor activator of nuclear factor $\mathrm{\kappa B}$ ligand.

of bone metabolism, and was affected by adipocytes in the bone marrow microenvironment. An increased RANKL/OPG ratio secreted by co-cultured osteoblasts compared with single-cultured was identified by ELISA analysis of cell culture medium (Table II). In addition, RANKL/OPG protein expression in osteoblasts was decreased in the co-culture group (Fig. 4A and B). RANK gene and protein expression in osteoclasts was increased by co-culture with adipocytes (Fig. 4A and B).

\section{Discussion}

The present study investigated the effect of adipocytes on preosteoblasts and preosteoclasts in the bone marrow microenvironment. Results demonstrate that i) bone mineral density (BMD) was significantly reduced in high-fat diet mice, ii) pre-osteoblast function was suppressed, iii) differentiation of pre-osteoclast was enhanced and iv) the RANKL/OPG ratio secreted by pre-osteoblasts was increased and RANK expression in pre-osteoclasts was increased by co-culture with adipocytes.

Adults with a high BMI have been previously reported to be associated with low BMD (10). Obesity is being increasingly recognized as a negative factor of bone metastasis in humans (11). In this study, high-fat diet mice exhibited high body weights. However, trabecular and cortical bone densities in the tibia were low. In addition, the number of adipocytes in bone marrow of high-fat diet mice was found to be significantly higher than that of the control. By contrast, a number of previous studies have observed that increased BMI is a positive factor on femur cortical bone mass due to its effect on leptin signaling (12). However, an increasing number of studies have indicated that obesity in female mice is accompanied by bone loss (8,13-15). This may be due to the role of adipose tissue as an endocrine organ, secreting pro-inflammatory cytokines, whose effects damage the trabecular bone (16). Therefore, the use of different mouse models in prevous studies may explain these inconsistencies.

Adipocytes derived from BMSCs undergo crosstalk with osteoblasts. In the present study, osteoblast expression of RUNX2, osteocalcin and ALP mRNA and protein was decreased in the late stages of differentiation following co-culture with adipocytes. It has previously been reported that the adipocyte-secreted hormone, adiponectin, may negatively modulate osteoblast function through adiponectinreceptor I-mediated changes in PPAR- $\gamma$ expression (17). The osteogenic function of osteoblasts was attenuated by adipocytes.

In this study, an osteoclastogenesis system was used to analyze RANKL-induced RAW 264.7 cells differentiated to osteoclastic cells exhibiting TRAP-positive staining (18). Osteoclastogenesis was found to be significantly increased by co-culture with adipocytes. However, the mechanism remains elusive. Oshima et al (19) demonstrated that adiponectin induced by cytokines secreted by adipocytes directly suppresses bone-resorption activity by inhibiting osteoclastogenesis. However, observations of Goto et al (20) demonstrate that primary human bone marrow adipocytes promote osteoclast differentiation and activities. Leptin, an adipocyte-derived cytokine, has been found to function as a negative regulator of bone mass in a mouse model (21).

The OPG/RANKL/RANK system in the bone microenvironment is an essential mechanism for regulation of bone metabolism. RANKL combined with RANK is associated with osteoclastogenesis through the c-Jun N-terminal kinase, nuclear factor $\kappa \mathrm{B}$ and protein kinase $\mathrm{B}$-mediated signaling pathways, which promote bone resorption. By contrast, RANKL combined with OPG inhibits osteoclast differentiation, which impairs bone resorption. The ratio of RANKL/RANK and RANKL/OPG regulates the bone microenvironment. If the balance between these ratios is disturbed, bone metabolism derangement is likely to occur (22).

The results of this study indicate that the OPG/RANKL ratio secreted by osteoblasts was decreased by adipocyte stimulation. As a result of reduced OPG/RANKL ratio, osteoclastogenesis was increased and was shown to be consistent with CTSK staining of bone sections from our animal model. In addition, osteoclast RANK expression was upregulated. Following this, adipocyte-secreted cytokines affected the OPG/RANKL/RANK trail system, resulting in a marked increase in osteoclast differentiation. Halade et al (16) reported that OPG secretion decreased and RANKL expression increased in osteoblasts stimulated with adipocyte-secreted factors. By contrast, Goto et al (20) demonstrated that adipocyte-secreted cytokines upregulated RANKL mRNA expression in osteoblasts, consistent with osteoclast differentiation in vitro. These observations are in agreement with those of this study and demonstrate that adipocytes increase osteoclastogenesis via the OPG/RANKL/RANK system in the bone microenvironment.

In conclusion, the present in vivo study has demonstrated that obesity caused by a high-fat diet results in significant bone resorption by increasing osteoclast and decreasing osteoblast functions. In addition, adipocyte-secreted cytokines were observed to attenuate osteoblast function and significantly increase osteoclastogenesis. The balance 
of the OPG/RANKL/RANK system was disrupted by adipocyte-derived cytokines. The results indicate that obesity is associated with decreases in bone density partly due to increased osteoclastogenesis.

\section{Acknowledgements}

The present study was supported by a research grant from the National Natural Sciences Research Program of China (no. 81070691).

\section{References}

1. Bahia L, Coutinho ES, Barufaldi LA, et al: The costs of overweight and obesity-related diseases in the Brazilian public health system: cross-sectional study. BMC Public Health 12: 440, 2012.

2. Mathus-Vliegen EM: Obesity and the elderly. J Clin Gastroenterol 46: $533-544,2012$

3. Cao JJ: Effects of obesity on bone metabolism. J Orthop Surg Res 6: 30, 2011.

4. Halade GV, Rahman MM, Williams PJ and Fernandes G: High fat diet-induced animal model of age-associated obesity and osteoporosis. J Nutr Biochem 21: 1162-1169, 2010.

5. Bredella MA, Torriani M, Ghomi RH, et al: Vertebral bone marrow fat is positively associated with visceral fat and inversely associated with IGF-1 in obese women. Obesity (Silver Spring) 19: 49-53, 2011.

6. Dimitri P, Bishop N, Walsh JS and Eastell R: Obesity is a risk factor for fracture in children but is protective against fracture in adults: a paradox. Bone 50: 457-466, 2012.

7. Prieto-Alhambra D, Premaor MO, Fina Aviles F, et al: The association between fracture and obesity is site-dependent: a population-based study in postmenopausal women. J Bone Miner Res 27: 294-300, 2012.

8. Kawai M, de Paula FJ and Rosen CJ: New insights into osteoporosis: the bone-fat connection. J Intern Med 272: 317-329, 2012

9. Aguila MB, Pinheiro Ada R, Parente LB and Mandarim-de-Lacerda CA: Dietary effect of different high-fat diet on rat liver stereology. Liver Int 23: 363-370, 2003.
10. Compston JE, Watts NB, Chapurlat R, et al: Obesity is not protective against fracture in postmenopausal women: GLOW. Am J Med 124: 1043-1050, 2011.

11. Shapses SA and Sukumar D: Bone metabolism in obesity and weight loss. Annu Rev Nutr 32: 287-309, 2012.

12. Thomas $T$ and Burguera B: Is leptin the link between fat and bone mass? J Bone Miner Res 17: 1563-1169, 2002.

13. Verzeletti GN, Gaio EJ, Linhares DS and Rösing CK: Effect of obesity on alveolar bone loss in experimental periodontitis in Wistar rats. J Appl Oral Sci 20: 218-221, 2012.

14. Dytfeld J, Ignaszak-Szczepaniak M, Gowin E, et al: Influence of lean and fat mass on bone mineral density (BMD) in postmenopausal women with osteoporosis. Arch Gerontol Geriatr 53: e237-e242, 2011.

15. Chen JR, Lazarenko OP, Wu X, et al: Obesity reduces bone density associated with activation of PPAR $\gamma$ and suppression of Wnt $/ \beta$-catenin in rapidly growing male rats. PLoS One 5: e13704, 2010

16. Halade GV, El Jamali A, Williams PJ, et al: Obesity-mediated inflammatory microenvironment stimulates osteoclastogenesis and bone loss in mice. Exp Gerontol 46: 43-52, 2011.

17. Savopoulos C, Dokos C, Kaiafa G and Hatzitolios A: Adipogenesis and osteoblastogenesis: trans-differentiation in the pathophysiology of bone disorders. Hippokratia 15: 18-21, 2011.

18. Hirotani H, Tuohy NA, Woo JT, et al: The calcineurin/nuclear factor of activated $\mathrm{T}$ cells signaling pathway regulates osteoclastogenesis in RAW264.7 cells. J Biol Chem 279: 13984-13992, 2004.

19. Oshima K, Nampei A, Matsuda M, et al: Adiponectin increases bone mass by suppressing osteoclast and activating osteoblast. Biochem Biophys Res Commun 331: 520-526, 2005.

20. Goto H, Osaki M, Fukushima T, et al: Human bone marrow adipocytes support dexamethasone-induced osteoclast differentiation and function through RANKL expression. Biomed Res 32: 37-44, 2011.

21. Fujita Y, Watanabe K and Maki K: Serum leptin levels negatively correlate with trabecular bone mineral density in high-fat diet-induced obesity mice. J Musculoskelet Neuronal Interact 12: 84-94, 2012.

22. Tyrovola JB, Spyropoulos MN, Makou M and Perrea D: Root resorption and the OPG/RANKL/RANK system: a mini review. J Oral Sci 50: 367-376, 2008. 